\title{
SMOOTH LIPSCHITZ RETRACTIONS OF STARLIKE BODIES ONTO THEIR BOUNDARIES IN INFINITE-DIMENSIONAL BANACH SPACES
}

\author{
DANIEL AZAGRA AND MANUEL CEPEDELLO BOISO
}

\author{
Abstract. Let $X$ be an infinite-dimensional Banach space and let $A$ be a $C^{p}$ \\ Lipschitz bounded starlike body (for instance the unit ball of a smooth norm). \\ We prove that \\ (1) The boundary $\partial A$ is $C^{p}$ Lipschitz contractible. \\ (2) There is a $C^{p}$ Lipschitz retraction from $A$ onto $\partial A$. \\ (3) There is a $C^{p}$ Lipschitz map $T: A \longrightarrow A$ with no approximate fixed points.
}

\section{INTRODUCTION AND MAIN RESULTS}

The well known Brouwer's fixed point theorem states that every continuous selfmap of the unit ball of a finite-dimensional Banach space admits a fixed point. This is equivalent to saying that there is no continuous retraction from the unit ball onto the unit sphere, or that the unit sphere is not contractible (the identity map on the sphere is not homotopic to a constant map). This result is no longer true in infinite dimensions (see [8]). In [14] B. Nowak showed that for several infinite-dimensional Banach spaces Brouwer's theorem fails even for Lipschitz mappings, and in [6] Y. Benyamini and Y. Sternfeld generalized Nowak's result for all infinite-dimensional normed spaces, establishing that for every infinite-dimensional space $(X,\|\cdot\|)$ there exists a Lipschitz retraction from the unit ball $B_{X}=\{x \in X:\|x\| \leq 1\}$ onto the sphere $S_{X}=\{x \in X:\|x\|=1\}$, and that $S_{X}$ is Lipschitz contractible.

In recent years a lot of work has been done on smoothness and Lipschitz properties in Banach spaces (see $[11,5]$ ). Following this trend it is natural to ask whether Nowak-Benyamini-Sternfeld's results can be sharpened so as to get $C^{p}$ smooth Lipschitz retractions of the unit ball onto the sphere of every infinite-dimensional Banach space whose norm is $C^{p}$ smooth. In this note we will show that this is indeed possible. In fact we generalize those results in two ways. Not only do they hold for the smooth category but also for a wider class of objects than balls and spheres: we show that for every infinite-dimensional Banach space with a $C^{p}$ Lipschitz bounded starlike body $A$ (where $p=0,1,2, \ldots, \infty$ ), there is a $C^{p}$ Lipschitz retraction of $A$ onto its boundary $\partial A$, and $\partial A$ is also $C^{p}$ Lipschitz contractible.

At this point we need to introduce some terminology. A closed subset $A$ of a Banach space $X$ is said to be a starlike body provided $A$ has a non-empty interior and there exists a point $x_{0} \in \operatorname{int} A$ such that each ray emanating from $x_{0}$ meets the boundary of $A$ at most once. In this case we will say that $A$ is starlike with respect to $x_{0}$. When dealing with starlike bodies, we can always assume that they are starlike

1991 Mathematics Subject Classification. Primary: 46B20. Secondary: 58B05, 46 T05. 
with respect to the origin (up to a suitable translation), and we will do so unless otherwise stated. For a starlike body $A$, we define the Minkowski functional of $A$ as

$$
q_{A}(x)=\inf \left\{\lambda>0 \mid \frac{1}{\lambda} x \in A\right\}
$$

for all $x \in X$. It is easily seen that for every starlike body $A$ its Minkowski functional $q_{A}$ is a continuous function which satisfies $q_{A}(r x)=r q_{A}(x)$ for every $r \geq 0$. Moreover, $A=\left\{x \in X \mid q_{A}(x) \leq 1\right\}$, and $\partial A=\left\{x \in X \mid q_{A}(x)=1\right\}$, where $\partial A$ stands for the boundary of $A$. Conversely, if $\psi: X \longrightarrow[0, \infty)$ is continuous and satisfies $\psi(\lambda x)=\lambda \psi(x)$ for all $\lambda \geq 0$, then $A_{\psi}=\{x \in X \mid \psi(x) \leq 1\}$ is a starlike body. Convex bodies (that is, closed convex sets with nonempty interior) are an important kind of starlike bodies. We will say that $A$ is a $C^{p}$ smooth (Lipschitz) starlike body provided its Minkowski functional $q_{A}$ is $C^{p}$ smooth (and Lipschitz) on the set $X \backslash q_{A}^{-1}(0)$.

Smooth starlike bodies are interesting because they are strongly related to bump functions and to $n$-homogeneous polynomials in Banach spaces (see [2] and [3]), therefore their geometrical properties are worth studying. It is worth noting that every Banach space having a $C^{p}$ smooth (Lipschitz) bump function has a $C^{p}$ smooth (Lipschitz) bounded starlike body too (and the converse is also true).

Before stating our main result we need a few topological definitions. Let $M, N$ be closed subsets of a Banach space $X$. We will say that two maps $f, g: M \longrightarrow N$ are $C^{p}$ Lipschitz homotopic provided there exist an open subset $U$ of $X$ containing $M$, an $\varepsilon>0$, and a $C^{p}$ smooth mapping $H:(-\varepsilon, 1+\varepsilon) \times U \longrightarrow X$ such that the restriction of $H$ to $[0,1] \times M$ is a Lipschitz homotopy joining $f$ to $g$, that is, $H:[0,1] \times M \longrightarrow N$ is Lipschitz continuous and satisfies $H(0, x)=f(x)$ and $H(1, x)=g(x)$ for all $x \in M$. Moreover we will demand that $H(t, x)=f(x)$ for $t \leq 0, x \in M$, and $H(t, x)=g(x)$ for $t \geq 1, x \in M$.

It is not difficult to see that, with this definition, 'being $C^{p}$ Lipschitz homotopic' endows the set of $C^{p}$ Lipschitz mappings from $M$ into $N$ with an equivalence relationship (one can join $C^{p}$ smooth homotopies without losing smoothness or Lipschitzness).

A closed subset $M$ of $X$ is said to be $C^{p}$ Lipschitz contractible if the identity map on $M$ is $C^{p}$ Lipschitz homotopic to a constant map on $M$. For instance, it is easy to check that every $C^{p}$ Lipschitz starlike body $A$ is $C^{p}$ Lipschitz contractible. It is also easy to see that every two maps on a $\left(C^{p}\right.$ Lipschitz) contractible set are always $\left(C^{p}\right.$ Lipschitz) homotopic (they are both homotopic to a constant).

Finally, we will say that $r: A \longrightarrow \partial A$ is a $C^{p}$ smooth Lipschitz retraction from the starlike body $A$ onto its boundary provided there exist an open subset $U$ of $X$ containing $A$ and a $C^{p}$ smooth mapping $R: U \longrightarrow X$ such that $R$ fixes all the points of $\partial A$, and the restriction of $R$ to $A$ is Lipschitz continuous and coincides with $r$.

Our main result is the following

Theorem 1.1. Let $X$ be an infinite-dimensional Banach space and let $A$ be a $C^{p}$ Lipschitz bounded starlike body. Then:

(1) The boundary $\partial A$ is $C^{p}$ Lipschitz contractible.

(2) There is a $C^{p}$ Lipschitz retraction from $A$ onto $\partial A$. 
(3) There is a $C^{p}$ Lipschitz map $T: A \longrightarrow A$ with no approximate fixed points, that is, $\inf \{\|x-T(x)\|: x \in A\}>0$.

As a corollary we obtain the following generalization of Benyamini-Sternfeld's theorem:

Corollary 1.2. Let $(X,\|\cdot\|)$ be an infinite-dimensional Banach space with an equivalent norm $\|\cdot\|$ which is $C^{p}$ smooth, and let $B_{X}$ and $S_{X}$ be its unit ball and unit sphere respectively. Then

(1) $S_{X}$ is $C^{p}$ Lipschitz contractible.

(2) There is a $C^{p}$ Lipschitz retraction of $B_{X}$ onto $S_{X}$.

(3) There is a $C^{p}$ Lipschitz map $T: B_{X} \longrightarrow B_{X}$ with no approximate fixed points.

If one is not interested in the Lipschitz property, it is a trivial consequence of the main result in [1] (see also [4]) that the sphere $S_{X}$ is $C^{p}$ contractible and there are $C^{p}$ smooth retractions from $B_{X}$ onto $S_{X}$. Unfortunately, the deleting diffeomorphisms obtained in $[1,4]$ are not Lipschitz, and corollary 1.2 cannot be deduced using those results. As a matter of fact, corollary 1.2 provides a new result even in the case $X=\ell_{2}$ with the usual hilbertian norm.

\section{THE PROOFS}

The proof of the main result is rather technical and will be split into three propositions and several lemmas. The general scheme of the proof follows that of [6], which in turn is a generalization with some modifications of Nowak's approach [14]. The proofs in $[6,14]$ are already involved in themselves and here they will be complicated with the difficulties peculiar to smooth maps and starlike bodies.

First of all it should be noted that parts (2) and (3) of theorem 1.1 are straightforward consequences of (1). Indeed, assume that $\partial A$ is $C^{p}$ Lipschitz contractible. Then there are an open subset $U$ of $X$ containing $\partial A$ and a $C^{p}$ smoth map $H$ : $(-\varepsilon, 1+\varepsilon) \times U \longrightarrow X$ such that the restriction of $H$ to $[0,1] \times \partial A$ is a Lipschitz homotopy joining the identity to a constant $x_{0}$ in $\partial A$. Without loss of generality we may assume that $H$ is defined on $(-\infty,+\infty) \times U$ and has the property that $H(t, x)=x_{0}$ for all $t \leq 0, x \in \partial A$, and $H(t, x)=x$ for all $t \geq 1, x \in \partial A$. Then the formula

$$
R(x)=H\left(2 \psi(x)-1, \frac{x}{\psi(x)}\right),
$$

where $\psi$ is the Minkowski functional of $A$, defines a $C^{p}$ smooth map on $X \backslash\{0\}$ with the property that $R(x)=x / \psi(x)$ whenever $\psi(x) \geq 1$ and $R(x)=x_{0}$ if $\psi(x) \leq \frac{1}{2}$. Then one can obviously extend $R$ (by putting $R(0)=x_{0}$ ) to a $C^{p}$ smooth map $R: X \longrightarrow X$ such that $R(x)=x$ whenever $\psi(x)=1$ and $R(x)=x_{0}$ for $\psi(x) \leq \frac{1}{2}$. The restriction of $R$ to the set $A=\{x \in X: \psi(x) \leq 1\}$ gives us a $C^{p}$ smooth retraction $r$ from $A$ onto its boundary. By using the fact that $H:[0,1] \times \partial A \longrightarrow \partial A$ is Lipschitz, it is easily seen that $r: A \longrightarrow \partial A$ is Lipschitz as well. This shows that part (1) of the theorem implies (2). On the other hand, once we have such a $C^{p}$ Lipschitz retraction $r$ one can easily get a $C^{p}$ Lipschitz map $T: A \longrightarrow A$ with no approximate fixed points: it is enough to take $T(x)=-r(x)$. 
Let us now start the proof of part (1) of 1.1. The following lemma tells us that for every two $C^{p}$ Lipschitz bounded starlike bodies $A_{1}, A_{2}$ the pair $\left(A_{1}, \partial A_{1}\right)$ is $C^{p}$ Lipschitz equivalent to the pair $\left(A_{2}, \partial A_{2}\right)$. We omit the proof of this result since it is an easy adaptation of that of Proposition 3 in [2].

Lemma 2.1. Let $X$ be a Banach space, and let $A_{1}, A_{2}$ be $C^{p}$ Lipschitz bounded starlike bodies. Then there exist a $C^{p}$ bi-Lipschitz diffeomorphism $g: X \longrightarrow X$ such that $g\left(A_{1}\right)=A_{2}, g\left(\partial A_{1}\right)=\partial A_{2}$, and $g(0)=0$. Moreover, $g(x)=\mu(x) x$, where $\mu: X \longrightarrow[0, \infty)$, and hence $g$ preserves the rays emanating from the origin.

Therefore, any $C^{p}$ Lipschitz property of a bounded starlike body or its boundary is shared with all the bounded starlike bodies and their boundaries. In particular the main theorem and all the auxiliary results which we will introduce in this section can be proved for any particular $C^{p}$ Lipschitz bounded starlike body in a Banach space $X$ and then, by using this lemma, extended for the rest of $C^{p}$ Lipschitz bounded starlike bodies, which are all equivalent. We will use this fact later on without further notice.

We will also need the following technical definition.

Definition 2.2. Let $X$ be a Banach space with a $C^{p}$ smooth Lipschitz bounded starlike body $A$, and let $\psi$ be its Minkowski functional. Let $M$ be a closed subset of $X, y_{0} \in M$, and $\varepsilon>0$. For every $y \in M, \delta>0$, define the pseudoball $B_{M}^{\psi}(y, \delta)=$ $\{x \in M: \psi(x-y) \leq \delta\}$.

A point $y_{0}$ is said to be an E-escaping point for $\psi$ in $M$ provided there exists a $C^{p}$ smooth Lipschitz mapping $T: M \longrightarrow M$ satisfying:

(1) $T$ is Lipschitz homotopic to the identity on $M$.

(2) $\inf \left\{\psi\left(T^{n} y_{0}-T^{m} y_{0}\right): n>m \geq 0\right\} \geq 10 \varepsilon$.

(3) For all $n \geq 0$, $T$ maps $B_{M}^{\psi}\left(T^{n} y_{0}, 2 \varepsilon\right)$ isometrically onto $B_{M}^{\psi}\left(T^{n+1} y_{0}, 2 \varepsilon\right)$ and, moreover, $T$ is merely a traslation when restricted to these sets.

(4) For all $n \geq 0, T^{-1}\left(B_{M}^{\psi}\left(T^{n+1} y_{0}, 2 \varepsilon\right)\right)=B_{M}^{\psi}\left(T^{n} y_{0}, 2 \varepsilon\right)$.

Now we state the three auxiliary propositions that we will use in the proof of the main theorem.

Proposition 2.3. Let $M, N$ be closed subsets of a Banach space $X$ which has a $C^{p}$ Lipschitz bounded starlike body A with Minkowski functional $\psi$. Suppose there is an E-escaping point $y_{0}$ in $M$. Let $g:[-1,1] \times M \longrightarrow N$ be a $C^{p}$ Lipschitz map which constantly attains the value $z_{0} \in N$ outside the set $\left[\frac{1}{4}, \frac{3}{4}\right] \times B_{M}^{\psi}\left(y_{0}, \varepsilon\right)$. Assume moreover that there exists an open subset $U$ of $X$ containing $M$ and an extension $\bar{g}:(-1-\varepsilon, 1+\varepsilon) \times U \longrightarrow X$ of $g$ such that $\bar{g}$ is $C^{p}$ smooth and satisfies $\bar{g}(t, x)=z_{0}$ for all $t \in(-1-\varepsilon, 1+\varepsilon)$ and $x \notin B_{U}^{\psi}\left(y_{0}, \varepsilon\right)$. Then $g$ is $C^{p}$ Lipschitz homotopic to the constant function $z_{0}$ in $[-1,1] \times M$ by means of a $C^{p}$ Lipschitz homotopy $H_{\tau}(t, x)$ $(0 \leq \tau \leq 1,(t, x) \in[-1,1] \times M)$ for which $H_{\tau}(t, x)=z_{0}$ whenever $|t| \geq \frac{3}{4}$.

Proposition 2.4. Let $X$ be an infinite-dimensional Banach space with a $C^{p}$ Lipschitz bounded starlike body. Then there exist $\varepsilon>0$ and another $C^{p}$ Lipschitz bounded symmetric starlike body $W$ such that its boundary $\partial W$ has an $\varepsilon$-escaping point with respect to $\psi=q_{W}$, the Minkowski functional of $W$. 
Proposition 2.5. Let $X$ be a Banach space and let $A$ be a $C^{p}$ Lipschitz starlike body which is bounded and symmetric, $x_{0} \in \partial A, \varepsilon>0$. Then the identity map on $\partial A$ is $C^{p}$ Lipschitz homotopic to a map $f: \partial A \longrightarrow \partial A$ which constantly attains the value $-x_{0}$ outside the set $\left\{x \in \partial A: \psi\left(x-x_{0}\right)<\varepsilon\right\}$ (where $\psi$ is the Minkowski functional of $A$ ). Moreover, $f$ can be assumed to have a $C^{p}$ smooth extension $\bar{f}: U \longrightarrow X$ (where $U$ is an open subset of $X$ containing $\partial A$ ) such that $\bar{f}(x)=-x_{0}$ whenever $\psi\left(x-x_{0}\right) \geq \varepsilon, x \in U$

\section{Proof of the theorem.}

Let $Y$ be a closed hyperplane of $X$. By Proposition 2.4 there is a $C^{p}$ Lipschitz bounded symmetric starlike body $W$ on $Y$ such that its boundary $\partial W$ admits an $\varepsilon$-escaping point $y_{0}$, for some $\varepsilon>0$ that can be assumed to satisfy $0<\varepsilon<\frac{1}{4}$. Let $q_{W}$ be the Minkowski functional of this starlike body. We may write $X=\mathbb{R} \times Y$. Now, let $V$ be a $C^{\infty}$ smooth Lipschitz bounded symmetric convex body of the plane $\mathbb{R}^{2}$ such that its boundary $\partial V$ contains the set

$$
\left\{(t, s) \in \mathbb{R}^{2}:|t| \leq 1,|s|=1\right\},
$$

and consider the Minkowski functional $q_{V}$ of $V$, which is a $C^{\infty}$ smooth equivalent norm on $\mathbb{R}^{2}$. Define now

$$
\psi(t, y)=q_{V}\left(t, q_{W}(y)\right)
$$

for every $(t, y) \in \mathbb{R} \times Y=X$. It is clear that $\psi$ is a $C^{p}$ Lipschitz function on $X \backslash\{0\}$ which is symmetric and positive homogeneous. Then

$$
U=\{(t, y) \in X: \psi(t, y) \leq 1\}
$$

is a $C^{p}$ Lipschitz bounded symmetric starlike body with the property that its boundary $\partial U$ contains the band $[-1,1] \times \partial W$. Without loss of generality we can assume that $U=A$ (see Lemma 2.1 and the preceding remarks), and it suffices to prove the theorem for this particular starlike body.

Next put $x_{0}=\left(\frac{1}{2}, y_{0}\right) \in \partial A$ and $z_{0}=-x_{0}$. By Proposition 2.5 there exists a $C^{p}$ Lipschitz map $f: \partial A \longrightarrow \partial A$ which is $C^{p}$ Lipschitz homotopic to the identity on $\partial A$, and which has a $C^{p}$ smooth extension $\bar{f}: U \longrightarrow X$ such that $\bar{f}(x)=$ $-x_{0}$ whenever $\psi\left(x-x_{0}\right) \geq \varepsilon, x \in U$. Note that if $x=(t, y) \in \partial A$ satisfies $\psi\left(x-x_{0}\right)<\varepsilon$ then, since $\varepsilon<\frac{1}{4}$, and taking into account the particular shape of $\partial A$, we have that $(t, y) \in\left[\frac{1}{4}, \frac{3}{4}\right] \times B_{\partial W}^{\psi}\left(y_{0}, \varepsilon\right) \subset[-1,1] \times \partial W$. Then it is clear that $g=f_{\mid[-1,1] \times \partial W}$ satisfies the conditions of Proposition 2.3 with $M=\partial W$ (bear in mind that $\bar{g}(t, y)=\bar{f}(t, y)=z_{0}$ whenever $\psi\left(0, y-y_{0}\right) \geq \varepsilon$ because the pseudoball $\left\{x \in X: \psi\left(x-x_{0}\right)<\varepsilon\right\}$ is contained in the cilynder $\left.\left\{(t, y) \in X: q_{W}\left(y-y_{0}\right)<\varepsilon\right\}\right)$. Since $y_{0}$ is an $\varepsilon$-escaping point in $\partial W$, it follows that $g$ is $C^{p}$ Lipschitz homotopic, as a map from $[-1,1] \times \partial W$ into $\partial A$, to the constant $z_{0}=-x_{0} \in \partial A$, by a $C^{p}$ Lipschitz homotopy $H_{\tau}(t, y)$ satisfying $H_{\tau}(t, y)=z_{0}$ whenever $|t| \geq \frac{3}{4}$.

Now, from the particular construction of $\partial A$, it is clear that one can extend $H_{\tau}$ to a $C^{p}$ Lipschitz homotopy $F_{\tau}$ by defining $F_{\tau}(x)=z_{0}$ for $x \in \partial A \backslash([-1,1] \times \partial W)$, and it is easily checked that $F_{\tau}$ is a $C^{p}$ Lipschitz homotopy joining $f$ to the constant $z_{0}$ in $\partial A$. Since $f$ is itself $C^{p}$ Lipschitz homotopic to the identity on $\partial A$, we can conclude that $\partial A$ is $C^{p}$ Lipschitz contractible to a point. 
Now we will give the proofs of Propositions 2.3, 2.4 and 2.5.

\section{Proof of Proposition 2.3.}

Let $T$ be the map associated to $\psi$ and the $\varepsilon$-escaping point $y_{0}$ in Definition 2.2. Let $\theta: \mathbb{R} \longrightarrow[0, \infty)$ be a $C^{p}$ Lipschitz mapping such that $\theta$ is strictly increasing in $(0, \infty), \theta(-t)=\theta(t), \theta(0)=0$, and $\theta(t)=|t|$ for $|t| \geq \frac{1}{8}$. Pick another nondecreasing $C^{p} \operatorname{map} \zeta: \mathbb{R} \longrightarrow \mathbb{R}$ such that $\zeta(t)=0$ for $t \leq \frac{1}{4}$ and $\zeta(t)=1$ for $t \geq \frac{3}{4}$. Now let us define two maps $f_{0}, f_{1}:[-1,1] \times M \longrightarrow N$ by

$$
f_{0}(t, x)= \begin{cases}g\left(\theta(t), T^{-n}(x)\right) & \text { whenever } t \geq 0, x \in B_{M}^{\psi}\left(T^{n}\left(y_{0}\right), \varepsilon\right), \text { and } n \geq 0 \\ g\left(\theta(t), T^{-n}(x)\right) & \text { whenever } t \leq 0, x \in B_{M}^{\psi}\left(T^{n}\left(y_{0}\right), \varepsilon\right), \text { and } n \geq 1 \\ z_{0} \quad \text { otherwise; } & \end{cases}
$$

and

$$
f_{1}(t, x)=\left\{\begin{array}{l}
g\left(\theta(t), T^{-n}(x)\right) \quad \text { whenever } t \geq 0, x \in B_{M}^{\psi}\left(T^{n}\left(y_{0}\right), \varepsilon\right), \text { and } n \geq 0 \\
z_{0} \text { otherwise. }
\end{array}\right.
$$

Note that on each "rectangle" $\left[\frac{1}{4}, \frac{3}{4}\right] \times B_{M}^{\psi}\left(T^{n}\left(y_{0}\right), \varepsilon\right)$ or $\left[-\frac{3}{4},-\frac{1}{4}\right] \times B_{M}^{\psi}\left(T^{n}\left(y_{0}\right), \varepsilon\right)$, $n \geq 0$, the mappings $f_{0}$ and $f_{1}$ are defined by the corresponding value (with respect to $T^{-n}$ ) of $g$ in the rectangle $\left[\frac{1}{4}, \frac{3}{4}\right] \times B_{M}^{\psi}\left(y_{0}, \varepsilon\right)$. All these rectangles are disjoint, by the definition of $\varepsilon$-escaping point. Since $T^{-n}$ is merely an affine traslation of $B_{M}^{\psi}\left(T^{n}\left(y_{0}\right), 2 \varepsilon\right)$ onto $B_{M}^{\psi}\left(y_{0}, 2 \varepsilon\right)$ and $g$ is $C^{p}$ smooth and Lipschitz, it is clear that the maps $f_{0}, f_{1}$ are $C^{p}$ and Lipschitz as well.

By assumption, $T$ is $C^{p}$ Lipschitz homotopic to the identity; let $G_{\tau}, \tau \in[0,1]$, be a $C^{p}$ Lipschitz homotopy joining the identity to $T$ in $M$. Then

$$
F_{\tau}(t, x)=\left\{\begin{array}{l}
f_{0}(t, x) \text { for } t \geq 0 \\
f_{0}\left(t, G_{\tau}(x)\right) \text { for } t \leq 0,
\end{array}\right.
$$

is a $C^{p}$ Lipschitz homotopy joining $f_{0}$ to $f_{1}$ in $[-1,1] \times M$. Now, the map $F_{\tau}^{1}(t, x)=$ $f_{1}(\theta(t)(1-\zeta(\tau))+\zeta(\tau), x)$ defines a $C^{p}$ Lipschitz homotopy joining $f_{1}$ to the constant $z_{0}$, and it is not difficult to see that the map

$$
F_{\tau}^{0}(t, x)=\left\{\begin{array}{l}
f_{0}(\theta(t) \zeta(\tau)+(1-\zeta(\tau)), x) \\
g(t, x) \text { for } x \in B_{M}^{\psi}\left(y_{0}, \varepsilon\right)
\end{array} \text { for } x \notin B_{M}^{\psi}\left(y_{0}, \varepsilon\right) ;\right.
$$

is a $C^{p}$ Lipschitz homotopy joining $g$ to $f_{0}$ (here we use the fact that $\bar{g}(t, x)=z_{0}$ whenever $\left.\psi\left(x-x_{0}\right) \geq \varepsilon, x \in U\right)$. We can then obtain the desired homotopy $H_{\tau}$ by applying successively $F_{\tau}^{0}, F_{\tau}$ and $F_{\tau}^{1}$. Since all of these homotopies have the constant value $z_{0}$ for $|t| \geq \frac{3}{4}$, the same is true of $H_{\tau}$.

In order to prove Proposition 2.4 a number of rather technical lemmas and facts will be required. Let us fix some standard notation used throughout these statements. If $K$ is a subset of $X$ and $x \in X$, we denote by $d_{\psi}(x, K):=\inf \{\psi(x-y)$ : $y \in K\}$. Also, the closed starlike body $B_{X}^{\psi}(x, r)=\{y \in X: \psi(y-x) \leq r\}$ will be simply written as $B^{\psi}(x, r)$.

The first technical tool we need is somehow a smooth version of Uryshon's lemma. 
Lemma 2.6. Let $X$ be a Banach space, and let $A$ be a $C^{p}$ Lipschitz bounded symmetric starlike body with Minkowski functional $\psi$ and $K$ be a compact subset of $X$. Then, for every $r>0$ there exists a $C^{p}$ Lipschitz function $f=f_{\psi, r, K}: X \longrightarrow[0,1]$ such that

(1) $f(x)=1$ whenever $d_{\psi}(x, K) \leq r / 2$, and

(2) $f(x)=0$ whenever $d_{\psi}(x, K) \geq r$.

Proof. Let $L_{\psi}$ be the Lipschitz constant of $\psi$ (i.e., $\psi(x)-\psi(y) \leq L_{\psi}\|x-y\|$, for all $x, y \in X)$. Since $K$ is compact there exist $x_{1}, \ldots, x_{l} \in K$ such that

$$
K \subset \bigcup_{j=1}^{l} B^{\|\cdot\|}\left(x_{j}, \frac{r}{4 L_{\psi}}\right) .
$$

Then pick a non-decreasing $C^{\infty}$ function $g: \mathbb{R} \longrightarrow[0,1]$ such that $g^{-1}(0)=\left(-\infty, \frac{3}{4} r\right]$ and $g^{-1}(1)=\left[\frac{7}{8} r, \infty\right)$. Put

$$
h(x)=\prod_{j=1}^{l} g\left(\psi\left(x-x_{j}\right)\right)
$$

for all $x \in X$. Since the functions $x \mapsto g\left(\psi\left(x-x_{j}\right)\right)$ are all bounded, Lipschitz and $C^{p}$, the function $h$, being a finite product of such functions, is also $C^{p}$ smooth and Lipschitz. Moreover, note that the Lipschitz constant of $h$ only depends on $\psi, r$ and the number of elements of the covering $(*)$.

By the construction of $h$ it is quite clear that $h(x)=1$ if $x \notin \cup_{j=1}^{l} B^{\psi}\left(x_{j}, \frac{7}{8} r\right)$, and therefore $h(x)=1$ whenever $d_{\psi}(x, K) \geq r$. Moreover, is easy to see that $h(x)=0$ if $x \in G:=\cup_{j=1}^{l} B^{\psi}\left(x_{j}, \frac{3}{4} r\right)$. Let us check that $G \supseteq\left\{x \in X: d_{\psi}(x, K) \leq r / 2\right\}$. In fact, if $x \in X$ is such that $d_{\psi}(x, K)=r / 2$, take $y \in K$ in such a way that $\psi(x-y)=r / 2$ and $x_{j}$ so that $y \in B^{\|\cdot\|}\left(x_{j}, \frac{r}{4 L_{\psi}}\right)$. Then it follows

$$
\psi\left(x-x_{j}\right) \leq \psi(x-y)+L_{\psi}\left\|y-x_{j}\right\| \leq \frac{3}{4} r .
$$

In order to conclude the proof it suffices to take $f(x)=1-h(x)$.

Fact 2.7. Let $X$ be a Banach space which has a $C^{p}$ Lipschitz bounded symmetric starlike body $A$ with Minkowski functional $\psi$ and $r>0$. Then for some $M>0$ one has that for every $a, b$ with $\|a\|=\|b\|=\frac{1}{4}$ there exists a $C^{p}$ Lipschitz function $f_{a, b}: X \longrightarrow[0,1]$ whose Lipschitz constant is less than or equal to $M$, and which satisfies that

(1) $f_{a, b}(x)=1$ whenever $d_{\psi}(x,[a, b]) \leq r / 2$, and

(2) $f_{a, b}(x)=0$ whenever $d_{\psi}(x,[a, b]) \geq r$.

Proof. Fix $r>0$. For every two arbitrary points $a, b$ of $X$ satisfying $\|a\|=\|b\|=\frac{1}{4}$, consider the compact set $K=[a, b]$. From Lemma 2.6, there exists a function $f_{a, b}$ that verifies conditions (1) and (2). We only have to ensure that the function $f_{a, b}$ constructed in the proof of Lemma 2.6 can be chosen with a Lipschitz constant that does not depend on the segment $[a, b]$. As we remarked before, the Lipschitz constant of $f_{a, b}$ only depends on the number of elements of the finite covering chosen in $(*)$. But, since the diameter of any segment $[a, b]$ is uniformly bounded, for every 
pair $a$ and $b$ it is easy to find an appropriate covering of $[a, b]$ with a fixed number of elements.

Lemma 2.8. Let $X$ be a Banach space which has a $C^{p}$ Lipschitz bounded symmetric starlike body $A$ with Minkowski functional $\psi$. Then for every $r>0$ there exists a constant $L>0$ so that for every $a, b \in X$ with $\|a\|=\|b\|=\frac{1}{4}$ there is a map $F=F_{a, b}: A \longrightarrow A$ satisfying

(1) $F$ is $C^{p}$ Lipschitz, and the Lipschitz constant of $F$ is less than or equal to $L$ (and therefore only depends on $\psi$ and $r$, but not on $a, b$ ).

(2) $F$ maps $B^{\psi}(a, r / 2)$ isometrically onto $B^{\psi}(b, r / 2)$; in fact $F$ is merely a translation when restricted to these sets, and $F(a)=b$.

(3) $F^{-1}\left(B^{\psi}(b, r / 2)\right)=B^{\psi}(a, r / 2)$.

(4) $F(x)=x$ whenever $d_{\psi}(x,[a, b]) \geq r$.

(5) F maps lines parallel to the segment $[a, b]$ into themselves.

Proof. For every such $a, b$ let us define $F=F_{a, b}: X \longrightarrow X$ by

$$
F(x)=x+f_{a, b}(x)(b-a)
$$

for all $x \in X$, where $f_{a, b}$ is the corresponding function obtained from fact 2.7. It is clear that $F$ is $C^{p}$ smooth and Lipschitz on $X$, with a Lipschitz constant not greater than $L=M+1$. Therefore $F$ satisfies condition (1) of the lemma.

It is evident from the definitions of $F$ and $f_{a, b}$ that $F$ satisfies properties (2), (4) and (5) as well. Let us see that $F$ satisfies property (3). If $F(x) \in B^{\psi}(b, r / 2)$ then

$$
\frac{r}{2} \geq \psi(b-F(x))=\psi\left(f_{a, b}(x) a+\left(1-f_{a, b}(x)\right) b-x\right) .
$$

Since $0 \leq f_{a, b}(x) \leq 1$ we have $f_{a, b}(x) a+\left(1-f_{a, b}(x)\right) b \in[a, b]$ and, therefore, it follows that $d_{\psi}(x,[a, b]) \leq r / 2$ and $f_{a, b}(x)=1$. Henceforth, we have

$$
\frac{r}{2} \geq \psi(b-F(x))=\psi(b-(x+(b-a))=\psi(a-x),
$$

which means that $x \in B^{\psi}(a, r / 2)$. This shows that $F^{-1}\left(B^{\psi}(b, r / 2)\right)=B^{\psi}(a, r / 2)$.

Lemma 2.9. Let $X$ be an infinite-dimensional Banach space which has a $C^{p}$ Lipschitz bounded symmetric starlike body A with Minkowski functional $\psi$. Then there exist some $\varepsilon>0$ and a point $x_{0}$ in the interior of $A$ which is an $\varepsilon$-escaping point in $A$ with respect to a map $T: A \longrightarrow A$ which in addition to properties (1)-(4) of Definition 2.2 satisfies $T(x)=x$ whenever $\psi(x) \geq \frac{3}{4}$.

Proof. Without loss of generality we can assume that $B_{X} \subseteq A$. Since $A$ is a bounded starlike body we know that there exists some $\alpha>0$ such that $\alpha\|x\| \leq \psi(x) \leq\|x\|$ for all $x \in X$. Note that no matter how $T$ is defined, $T$ will be $C^{p}$ Lipschitz homotopic to the identity on $A$ because $A$ is starlike and hence $C^{p}$ Lipschitz contractible (so that both $T$ and the identity are homotopic to a constant on $A$ ).

Let $\left(w_{n}\right)_{n \in \mathbb{N}}$ be a normalized basic sequence in $X$ with biorthogonal functionals $\left(w_{n}^{*}\right)_{n \in \mathbb{N}} \subset X^{*}$ satisfying $\left\|w_{n}^{*}\right\| \leq 4$ (one can always take such a sequence, see [10], p. 93), and put $z_{n}=\frac{1}{4} w_{n}$ for all $n \in \mathbb{N}$. Let us denote by $L_{n, k}$ the straight line $\left\{t z_{n}+(1-t) z_{k}: t \in \mathbb{R}\right\}$ passing through $z_{n}$ and $z_{k}($ for $n \neq k)$. It is easy to see 
that, if $\{n, k\} \cap\{m, l\}=\emptyset$ then $\|x-y\| \geq \frac{1}{32}$ for all $x \in L_{n, k}, y \in L_{m, l}$. This implies that $\psi(x-y) \geq \frac{\alpha}{32}$ for all $x \in L_{n, k}, y \in L_{m, l}$; that is, $d_{\psi}\left(L_{n, k}, L_{m, l}\right) \geq \frac{\alpha}{32}$.

Now take $r=\frac{\alpha}{320}$, and for every $n, k \in \mathbb{N}, n \neq k$, pick a function $F_{n, k}: A \longrightarrow A$ satisfying the conditions of Lemma 2.8 for $a=z_{n}$ and $b=z_{k}$, and put $\varepsilon=r / 4$. For this choice of $\varepsilon$ and $r$ we have

$$
d_{\psi}\left(L_{n, k}, L_{m, l}\right) \geq \frac{\alpha}{32} \geq 10 r>20 \varepsilon .
$$

Note that, by this inequality and the construction of $F$, if $\{n, k\} \cap\{m, l\}=\emptyset$ then $F_{n, k}(x)=x$ whenever $d_{\psi}\left(x, L_{m, l}\right) \leq r=4 \varepsilon$, and in particular whenever $F_{m, l}(x) \neq x$ or $x=F_{m, l}(y)$ for some $y \neq x$. Then the infinite composition

$$
V_{1}(x)=\left(\cdots \circ F_{2 n-1,2 n} \circ \cdots \circ F_{3,4} \circ F_{1,2}\right)(x)
$$

is well defined and satisfies

(1) $V_{1}$ is Lipschitz. Indeed, take into account that all the maps $F_{n, k}$ involved in the definition of $V_{1}$ have a Lipschitz constant which is less than or equal to a fixed constant $L$, and the infinite composition defining $V_{1}$ is uniformly locally finite. In fact for every $x \in A$ there exists a neighbourhood of $x$ in $A$ such that $V_{1}$ coincides with one of the $F_{n, k}$ when restricted to this neighbourhood. From these properties and from the facts that $A$ contains the unit ball $B_{X}$, which is a convex set, and $V_{1}$ obviously restricts to the identity outside $B_{X}$, one can easily deduce that $V_{1}$ is Lipschitz (with a Lipschitz constant less than or equal to $L$ ) on $A$.

(2) $V_{1}$ is $C^{p}$ smooth (this is again a consequence of the fact that the infinite composition defining $V_{1}$ is locally finite and all the functions $F_{n, k}$ are $C^{p}$ smooth).

(3) $V_{1}$ maps $B^{\psi}\left(z_{2 n-1}, 2 \varepsilon\right)$ isometrically (in fact it is merely a translation when restricted to this set) onto $B^{\psi}\left(z_{2 n}, 2 \varepsilon\right)$.

(4) $V_{1}^{-1}\left(B^{\psi}\left(z_{2 n}, 2 \varepsilon\right)\right)=B^{\psi}\left(z_{2 n-1}, 2 \varepsilon\right)$.

(5) $V_{1}(x)=x$ whenever $\psi(x) \geq \frac{3}{4}$.

Let us define as well

$$
V_{2}(x)=\left(\cdots \circ F_{2 n, 2 n+1} \circ \cdots \circ F_{4,5} \circ F_{2,3}\right)(x) .
$$

Then $V_{2}$ is also a $C^{p}$ Lipschitz map that satisfies

(3') $V_{2}$ maps $B^{\psi}\left(z_{2 n}, 2 \varepsilon\right)$ isometrically (in fact it is a translation when restricted to this set) onto $B^{\psi}\left(z_{2 n+1}, 2 \varepsilon\right)$.

(4') $V_{2}^{-1}\left(B^{\psi}\left(z_{2 n+1}, 2 \varepsilon\right)\right)=B^{\psi}\left(z_{2 n}, 2 \varepsilon\right)$.

(5') $V_{2}(x)=x$ whenever $\psi(x) \geq \frac{3}{4}$.

Now let us define $T=V_{2} \circ V_{1}$. It is clear that $T$ is a $C^{p}$ Lipschitz map. It only remains to check that $z_{1}$ is an $\varepsilon$-escaping point for $T$. Indeed, as said above, condition (1) of Definition 2.2 is trivially satisfied. It is also clear that $T^{n} z_{1}=z_{2 n+1}$, and condition (2) of 2.2 follows from (**) above. Finally, conditions (3) and (4) of 2.2 follow respectively from $\left(3,3^{\prime}\right)$ and $\left(4,4^{\prime}\right)$ above.

\section{Proof of Proposition 2.4.}


Let $Y$ be a closed hyperplane of $X$ and identify $X=Y \times \mathbb{R}$. Let $A$ be a $C^{p}$ Lipschitz bounded symmetric starlike body in $Y$ with Minkowski functional $q_{A}$. By Lemma 2.9 there exist $\varepsilon>0$ and $y_{0} \in A$ with $q_{A}\left(y_{0}\right)<\frac{3}{4}$ such that $y_{0}$ is an $\varepsilon$ escaping point with respect to a map $V: A \longrightarrow A$ which satisfies $V(x)=x$ for $q_{A}(x) \geq \frac{3}{4}$. Take a $C^{\infty}$ Lipschitz convex body $U$ on the plane $\mathbb{R}^{2}$ such that its boundary $\partial U$ contains the set

$$
\left\{(s, t):|s|=1,|t| \leq \frac{3}{4}\right\} \cup\left\{(s, t):|s| \leq \frac{3}{4},|t|=1\right\},
$$

and consider the Minkowski functional of $U, q_{U}$, which is $C^{p}$ and Lipschitz. Define now

$$
\psi(y, t)=q_{U}\left(q_{A}(y), t\right)
$$

for every $(y, t) \in Y \times \mathbb{R}=X$. It is clear that $\psi$ is a $C^{p}$ Lipschitz function on $X \backslash\{0\}$ which is symmetric and positive homogeneous. Then $W=\{(y, t) \in X: \psi(y, t) \leq$ $1\}$ is a $C^{p}$ Lipschitz bounded symmetric starlike body with the property that its boundary $\partial W$ contains the set

$$
\left\{(y, t) \in X: q_{A}(y)=1,|t| \leq \frac{3}{4}\right\} \cup\left\{(y, t) \in X: q_{A}(y) \leq \frac{3}{4},|t|=1\right\} .
$$

Now define $T: \partial W \longrightarrow \partial W$ by

$$
T(y, t)=\left\{\begin{array}{l}
(V(y), 1) \text { if } t=1 \\
(y, t) \text { if } t \neq 1
\end{array}\right.
$$

It is clear that $T$ is a well-defined Lipschitz map. Moreover $T$ is $C^{p}$ smooth on $\partial W$ because $T$ can be extended to a map $\bar{T}: X \longrightarrow X$ which is $C^{p}$ smooth (indeed, $\bar{T}(y, t)=\left(\frac{1}{2}(t+1) V(y)+\frac{1}{2}(1-t) y, t\right)$ does the job; note that if $(y, t) \in \partial W$ and $|t| \neq 1$ then $q_{A}(y) \geq \frac{3}{4}$ and therefore $V(y)=y$; this implies that $\left.\bar{T}_{\left.\right|_{\partial W}}=T\right)$.

Let us check that $\left(y_{0}, 1\right)$ is an $\varepsilon$-escaping point of $\partial W$ associated with this map $T$. Conditions (2), (3) and (4) of Definition 2.2 follow directly from the corresponding properties of $V$ (take into account that $\psi(y, 0)=q_{A}(y)$ for all $y \in Y$ ). It only remains to prove that $T$ is $C^{p}$ Lipschitz homotopic to the identity on $\partial W$. Take a $C^{\infty}$ smooth function $\theta: \mathbb{R} \longrightarrow \mathbb{R}$ such that $\theta(t)=0$ for $t \leq \frac{1}{4}$ and $\theta(t)=1$ for $t \geq \frac{3}{4}$, and define $H:[0,1] \times \partial W \longrightarrow \partial W$ by

$$
H_{\tau}(x)=H_{\tau}(y, t)=\frac{\theta(\tau) T(x)+(1-\theta(\tau)) x}{\psi(\theta(\tau) T(x)+(1-\theta(\tau)) x)} .
$$

Note that, since $T(x) \neq x$ only for points $x=(y, t)$ with $t=1$ and $q_{A}(y) \leq \frac{3}{4}$, we have $\psi(\tau T(x)+(1-\tau) x) \geq 1$ for all $x, \tau$, so that $H$ is clearly well defined and Lipschitz on $[0,1] \times \partial W$. It is also clear that $H$ is a homotopy joining $T$ to the identity on $\partial W$. Moreover, $H$ is $C^{p}$ smooth because it can be extended to a $C^{p}$ defined on an open neighbourhood of $[0,1] \times \partial W$ (indeed, it is enough to consider

$$
H_{\tau}(x)=H_{\tau}(y, t)=\frac{\theta(\tau)\left(\frac{1}{2}(t+1) V(y)+\frac{1}{2}(1-t) y, t\right)+(1-\theta(\tau))(y, t)}{\psi\left(\theta(\tau)\left(\frac{1}{2}(t+1) V(y)+\frac{1}{2}(1-t) y, t\right)+(1-\theta(\tau))(y, t)\right)},
$$

which is $C^{p}$ smooth on the subset of points of $\mathbb{R} \times X$ for which the denominator of this quotient is not zero, an open neighbourhood of $[0,1] \times \partial W)$. 


\section{Proof of Proposition 2.5.}

Let $\theta: \mathbb{R} \longrightarrow[0,1]$ be a non-increasing $C^{\infty}$ smooth function such that $\theta(t)=1$ for $t \leq \varepsilon / 2$ and $\theta(t)=0$ for $t \geq \varepsilon$. Let us define

$$
\bar{f}(x)=\frac{\theta\left(\psi\left(x-x_{0}\right)\right) x-\left(1-\theta\left(\psi\left(x-x_{0}\right)\right)\right) x_{0}}{\psi\left(\theta\left(\psi\left(x-x_{0}\right)\right) x-\left(1-\theta\left(\psi\left(x-x_{0}\right)\right)\right) x_{0}\right)}
$$

for every $x$ such that the above denominator is not zero. It is clear that $\bar{f}$ is a $C^{p}$ smooth map with the property that $\bar{f}(x)=-x_{0}$ whenever $\psi\left(x-x_{0}\right) \geq \varepsilon$. The restriction of $\bar{f}$ to $\partial A$ gives us a $C^{p}$ smooth Lipschitz function $f: \partial A \longrightarrow \partial A$ which constantly attains the value $-x_{0}$ outside $\left\{x \in \partial A: \psi\left(x-x_{0}\right)<\varepsilon\right\}$. To see that $f$ is Lipschitz it is enough to take into account that there is some $\delta>0$ such that

$$
\left\|\theta\left(\psi\left(x-x_{0}\right)\right) x-\left(1-\theta\left(\psi\left(x-x_{0}\right)\right)\right) x_{0}\right\| \geq \delta
$$

for all $x \in \partial A$, and the maps $\theta, \psi$ are Lipschitz. Finally, define $H:[0,1] \times \partial A \longrightarrow \partial A$ by

$$
H(\tau, x)=\frac{(1-\theta(\tau)) x+\theta(\tau) f(x)}{\psi((1-\theta(\tau)) x+\theta(\tau) f(x))} .
$$

It is not difficult to see that $H$ is a $C^{p}$ Lipschitz homotopy joining the identity to the function $f$ on $\partial A$.

\section{Acknowledgements}

This research was carried out during a postdoctoral stay of the first-named author in the Equipe d'Analyse de l'Université Pierre et Marie Curie, Paris 6. This author is indebted to the Equipe d'Analyse and very especially to Gilles Godefroy for their kind hospitality and generous advice.

\section{REFERENCES}

[1] D. Azagra, Diffeomorphisms between spheres and hyperplanes in infinite-dimensional Banach spaces, Studia Math. 125 (2) (1997), p. 179-186.

[2] D. Azagra, On the topological classification of starlike bodies in Banach spaces, preprint, 1999.

[3] D. Azagra and R. Deville Starlike bodies and bump functions in Banach spaces, preprint, 1999.

[4] D. Azagra and T. Dobrowolski, Smooth negligibility of compact sets in infinite-dimensional Banach spaces, with applications, Math. Ann. 312 (1998), no. 3, 445-463.

[5] Y. Benyamini and J. Lindenstrauss, Geometrical Nonlinear Functional Analysis, Volume I, to appear.

[6] Y. Benyamini and Y. Sternfeld, Spheres in infinite-dimensional normed spaces are Lipschitz contractible, Proc. Amer. Math. Soc. 88, n. 3 (1983), 439-445.

[7] C. Bessaga, Every infinite-dimensional Hilbert space is diffeomorphic with its unit sphere, Bull. Acad. Polon. Sci., Sér. Sci. Math. Astr. et Phys.14 (1966), pp. 27-31.

[8] C. Bessaga and A. Pelczynski, Selected topics in infinite-dimensional topology, Monografie Matematyczne, Warszawa 1975.

[9] H. Cartan, Calcul différentiel, Hermann, Paris 1967.

[10] M. M. Day, Normed linear spaces, third ed., Springer-Verlag, Berlin, 1973.

[11] R. Deville, G. Godefroy, and V. Zizler, Smoothness and renormings in Banach spaces, vol. 64, Pitman Monographies and Surveys in Pure and Applied Mathematics, 1993.

[12] K. Goebel, On the minimal displacement of points under Lipschitzian mappings, Pacific J. Math. 45, n.1 (1973), 151-163. 
[13] K. Goebel and W. A. Kirk, A fixed point theorem for transformations whose iterates have uniform Lipschitz constant, Studia Math. 47 (1973), 135-140.

[14] B. Nowak, On the Lipschitzian retraction of the unit ball in infinite-dimensional Banach spaces onto its boundary, Bull. Acad. Polon. Sci. 27 (1979), 861-864.

Departamento de Análisis Matemático. Facultad de Ciencias Matemáticas. Universidad Complutense. 28040 Madrid, SPAIN

Equipe d'Analyse, Université Pierre et Marie Curie-Paris 6. 4, place Jussieu, 75005 Paris, FRANCE.

Departamento de Análisis Matemático. Universidad de Sevilla. Sevilla, SPAIN.

E-mail addresses: daniel@sunam1.mat.ucm.es, boiso@cica.es 